\title{
Handoff Mechanism using Spectrum Pool
}

\author{
Shubhangi Mahamuni \\ Assistant Professor, MAE, \\ Alandi(D) \\ Pune, Maharashtra, India
}

\author{
Vivekanand Mishra, PhD. \\ (IEEE senior member) \\ Associate professor, SVNIT, \\ Surat, Gujrat, India
}

\author{
Angela Amphewan, \\ School of computing University \\ Utara Malaysia, 06010 Sintok, \\ Malaysia
}

\begin{abstract}
In this paper, spectrum pool is one of the efficient techniques for spectrum sensing selection. Cognitive radio always preferred for accessing free channels efficiently with minimum handoff delay. The performance of spectrum pool has been implemented and compared with conventional handoff mechanism. Spectrum handoff occurs when primary user appears and the secondary users using this particular licensed channel. This particular spectrum handoff would use channels for the handoff process from the spectrum pool formed by proposed method. Spectrum sensing plays important role in case of cognitive radio. For accessing the spectrum reuse functionality, secondary users used to sense the radio frequency environment. For this propose we proposed two different modules one is spectrum pool and other will be secondary user with multiple antennas.
\end{abstract}

\section{Keywords}

Cognitive Radio, Spectrum pool, Spectrum Handoff.

\section{INTRODUCTION}

Spectrum pool varies from traditional handoff in wireless networks. Spectrum handoff takes place upon PU arrival whereas the handoff in wireless networks takes place due to signal degradation and user mobility. In nearly all of the existing spectrum handoff schemes [10]-[17], the handoff performance has been examined using numerous methods, taking into account different aspects discussed as follows: In [10], authors explored spectrum handoff for link maintenance of three types, i.e., non-spectrum handoff, the proactive spectrum handoff, and the spectrum handoff depending on sensing mechanism. The authors have observed the performance based on the probability of link maintenance as well as the effective data rate of the SU's transmission. However their results reveal that there could be chances of erroneous and incorrect channel selection hence affecting the performance of SU. The authors in [11-18] have measured the handoff performance in opportunistic1 and negotiated2 situations. They have generalized the key teletraffic parameters in both the primary system and the secondary system.

\section{RELATED WORK}

The detail information about the cognitive radio was found [1]. The information of the spectrum band which is assigned by Federal Communications Commission (FCC) found the licensed band allocated to TV channels highly underutilized. To improve spectrum efficiency, they permitted secondary systems to function in the frequency band allocated to the television services [2] [3] [4]. In order to utilize the spectrum very efficiently, the IEEE 802.22 working Group (WG) developed WRAN (Wireless Regional Area Network), a secondary system that will be operating in the licensed TV channels [5] [6].The WRAN system was developed to provide wireless broadband access to the rural areas where broadband services have not yet reached due to certain physical limitations. To achieve this purpose, CR is seen as the solution, allowing capable and reliable use of spectrum by adjusting to the radio's environment accordingly [1] [7]. CR has emerged as a potential technology in order to increase the usage of the limited radio bandwidth in addition with accommodating the growing number of wireless services, devices and networks. A CR transceiver is an intelligent device that adjusts itself to the radio environment consequently increasing the utilization of the limited radio resources while providing flexibility in wireless access [8]. Although the requirement of spectrum sensing has been eliminated recently by FCC [2] as CR will now be equipped with TV channel database, the traditional handoff mechanism was with the conventional method which consists of number if SUs continuously searching for the free channel. This paper focuses on the issue of handoff delay caused during spectrum mobility under the new FCC September 2010 release. Radio frequency spectrum is a very precious and valuable resource. According to [9], TV channels and their guard channels are to be used for communication in IEEE 802.22, which is the first standard implementing CR technology. Different schemes used for the spectrum handoff mechanism has been explained [10-18].The rest of the paper is organized as follows: In section 2 and 3 related work and spectrum handoff mechanism is discussed. Followed by section 4 and 5 which presents the proposed handoff with the formation of a spectrum pool for the real time handoff mechanism and simulation results.

\section{THE SPECTRUM POOL SYSTEM}

Spectrum pool formation consists of formation of spectrum holes for a secondary user PUs with spectrum holes 1 or 0 indicated that the PU has taken up hole or not as shown in fig.1. In [8] formed a spectrum pool for a single secondary user only but we designed it for multiple secondary users. Let pool contains $(\mathrm{K}=\mathrm{N})$ holes. It maintains sensing results in every slot. For the formation of the spectrum pool the parameters used are $\mathrm{i}, \operatorname{Ri}(\mathrm{t}), \operatorname{Si}(\mathrm{t})$ where $\mathrm{i}$ indicates the channel state whether it is busy or idle, $\mathrm{s}(\mathrm{t})$ is the sensing time of a particular channel, Ri(t) indicates busy period of a particular channel. In our system sensing time is 10 seconds .After every $10 \mathrm{sec}$ spectrum pool updates the position of a channel .For choosing a spectrum hole from a spectrum pool secondary user will be responsible. It will select a channel according to the information of a spectrum pool. 


\section{PU-primary User SU-secondary User PBS-Primary Base station}

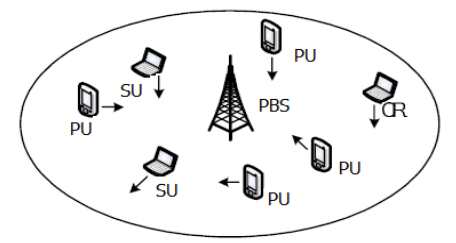

Fig.1 CR spectrum Sensing Scinario

Initially in one slot, the second receiver must choose one spectrum hole from the pool, apart from the band in use on the basis of our spectrum pool as shown in fig.1 Its duty is to give the SU the state of all bands. So it will choose the spectrum band with the largest necessity $\operatorname{Ri}(\mathrm{t})$ as follows.

$$
\operatorname{si}(t)=\sum R i(t)
$$

where si(t) $s$ the band index to sense in time $t$ and ioi $s$ the inuse band index. When it's time to access another spectrum band because of the primary's return, it also lookups the spectrum pool. For the Ri(t), the smaller the better, in the case that the band state is idle. But we couldn't ignore the possibility that the idle state comes up after a long white time. Therefore, the method to choose the access band can be drawn out as follows

$a(t)=\max \operatorname{si}(t)$

We have considered here for the formation of a spectrum pool 20 channels. The status of the band depending upon the utilization as shown in fig. 2 has been decided. If the spectrum hole is busy indicated by 1 and which was free considered as o. After some time the renewal of the spectrum pool takes place. In our simulation initially channel 1,10 and 12 were 1.After renewal band 10 was 0 , which clearly indicates that the status of channel utilization has been improved. Here we had calculated end to end delay after formation of spectrum pool of cognitive radio Ad-hoc Network and compared with the conventional cognitive radio Ad-hoc network.

\section{Performance Analysis:}

For simulation NS-2 software is used. In order to compare our results with the conventional spectrum

Fig.2 Spectrum Band Sensing In a Cognitive Radio Ad-hoc Network

\footnotetext{
Ele Edit Wiew Ierminal Tabs Help

Channel: 1 Channel: 1 Channel: 1 Channel: 1 Channel: 1 Channel: 1 Channel: 1 Cha 2

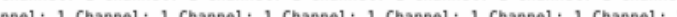

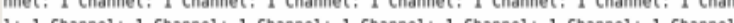

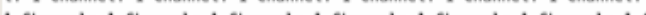
1 Channet. 1 Channet. 1 Channet. 1 Channet. 1 Channet. 1 Channet: 1 Channel: 1 hannel: 1 Channel: 1 Channel: 1 Channel: 1 Channel: 1 Channel: 1 Channel: 1 Cha nel: 1 Channel: 1 Channel: 1 Channel: 1 Channel: 1 Channel: 1 Channel: 1 Channe : 1 Channel: 1 Channel: 1 Channel: 1 Channel: 1 Channel: 1 Channel: 1 Channel: Channel: 1 Channel: 1 Channel: 1 Channel: 1 Channel: 1 Channel: 1 Channel: 1 ch annel: 1 Channel: 1 Channel: 1 Channel: 1 Channel: 1 Channel: 1 Channel: 1 Chann el: $\theta$ Channel: 1 Channel: 1 Channel: $\theta$ Channel: 1 Channel: $\theta$ Channel: 1 Channel: A Channel: $\theta$ Channel: I Channel: 1 Channel: 1 Channel: 1 Channel: 1 Channel: 1 Chane 1: 1 Channel: 1 Chane 1: 1 Channel: 1 Channel: 1 Cnannel: 1 Chanel: 1 cha

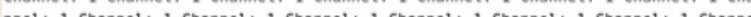

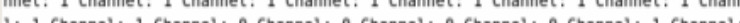
t: 1 Channet: 1 Channet: $\theta$ Channet: $\theta$ Channet: $\theta$ Channet: $\theta$ Channet: 1 Channel 1 Channel: 1 Channel: 1 Channel: 1 Channel: 1 Channel: 1 Channel: 1 Channel: 1 hannel: 1 Channel: 1 Channel: 1 Channel: 1 Channel: 1 Channel: $\theta$ Channel: 2 Chan nel: 2 Channel: 2 Channel: 2 Channel: 2 Channel: $\theta$ Channel: $\theta$ Channel: 1 Channe I Channel: 1 Channel: 1 Channel: 1 Channel: 1 Channel: 1 Channel: 1 Channel: Channel: 1 Channel: 1 Channel: 1 Channel: 1 Channel: 1 Channel: 1 Channel: 1 on annel: $\theta$ Channel: 1 Channel: 1 Channel: 1 Channel: 1 Channel: 1 Channel: 1 Chan el: 1 Channel: $\theta$ Channel: 1 Channel: 1 Channel: 1 Channel: 1 Channel: 1 Channel: 1 channel: 1 channel: 0 chane $1:-1$ chanel: 1 channel: 1 channel: 1 channel:

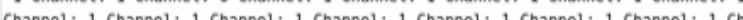

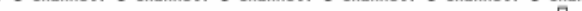
nnel: 1 Channel: 1 Channel: 1 Channel: 1 [root@localhost ns-2.31] \#]
}

Table I. Formation of a spectrum pool before pool renewal and after pool renewal based on the spectrum pool

\begin{tabular}{|l|l|l|l|l|l|}
\hline Sr.No & $\begin{array}{l}\text { Channel } \\
\text { No. }\end{array}$ & $\begin{array}{l}\text { Status } \\
\text { of the } \\
\text { Channel } \\
\text { Before } \\
\text { Renewal }\end{array}$ & Sr.No & $\begin{array}{l}\text { Channel } \\
\text { No. }\end{array}$ & $\begin{array}{l}\text { Status } \\
\text { of the } \\
\text { Channel } \\
\text { After } \\
\text { Renewal }\end{array}$ \\
\hline 0 & 0 & 0 & 0 & 0 & 0 \\
\hline 1 & 1 & 1 & 1 & 1 & 1 \\
\hline 2 & 2 & 0 & 2 & 2 & 0 \\
\hline 3 & 3 & 0 & 3 & 3 & 0 \\
\hline 4 & 4 & 0 & 4 & 4 & 0 \\
\hline 5 & 5 & 0 & 5 & 5 & 0 \\
\hline 6 & 6 & 0 & 6 & 6 & 0 \\
\hline 7 & 7 & 0 & 7 & 7 & 0 \\
\hline 8 & 8 & 0 & 8 & 8 & 0 \\
\hline 9 & 9 & 0 & 9 & 9 & 0 \\
\hline 10 & 10 & 1 & 10 & 10 & 0 \\
\hline 11 & 11 & 0 & 11 & 11 & 0 \\
\hline 12 & 12 & 1 & 12 & 12 & 1 \\
\hline 13 & 13 & 0 & 13 & 13 & 0 \\
\hline 14 & 14 & 0 & 14 & 14 & 0 \\
\hline 15 & 15 & 0 & 15 & 15 & 0 \\
\hline 16 & 16 & 0 & 16 & 16 & 0 \\
\hline 17 & 17 & 0 & 17 & 17 & 0 \\
\hline 18 & 18 & 0 & 18 & 18 & 0 \\
\hline 19 & 19 & 0 & 19 & 19 & 0 \\
\hline & 19 & 0 & 0 & & \\
\hline
\end{tabular}

The SU has exponentially distributed packet length (bs(x)) defined in [13] as, where $\mu$ on is the packet inter arrival time of SU. $\mu_{o}$ is the packet inter-arrival time of PU. The figure shows that with greater $\mu \mathrm{s}$, the total service time is considerably less.

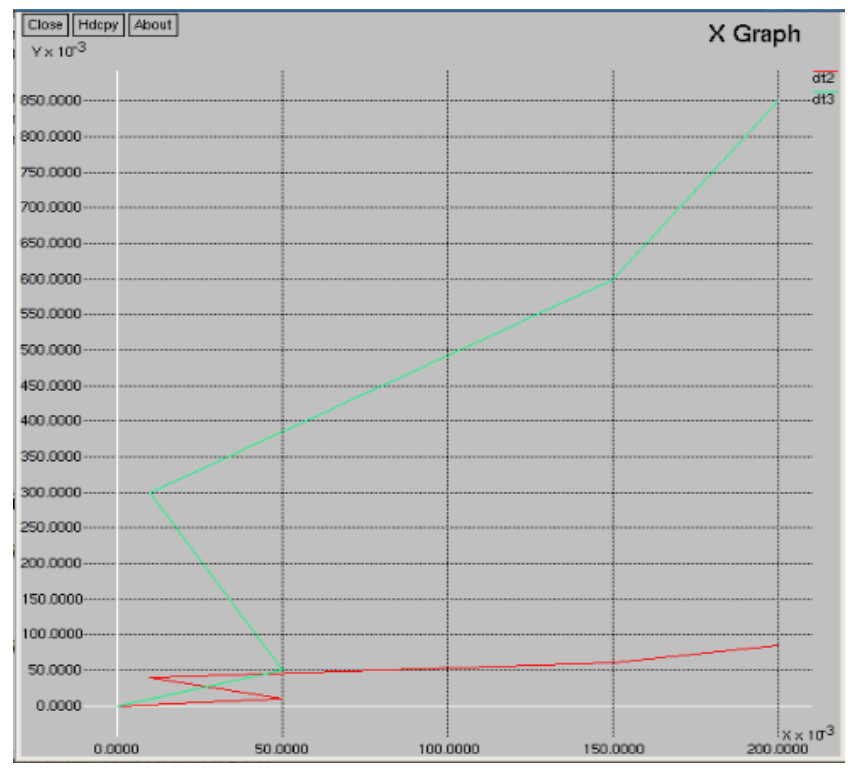

Fig.3.Transmission Time With/Without Spectrum pool 


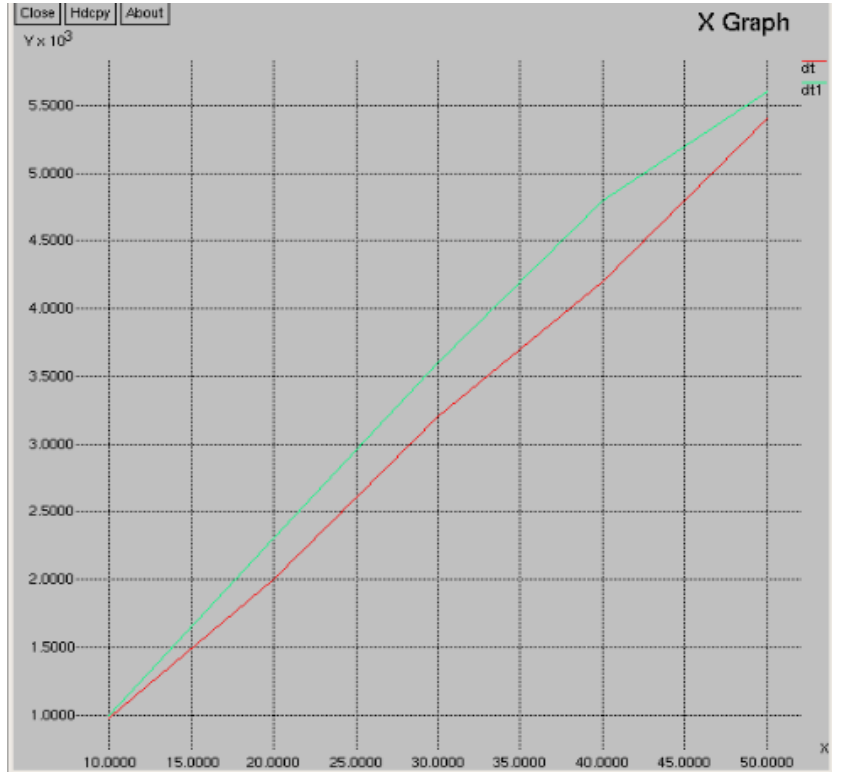

Fig.4. Handoff times with/without spectrum pool

\section{Conclusions}

In this paper Packet based handoff mechanism for minimization of spectrum handoff delay is suggested that makes effective use of the packet based channels for communication in $\mathrm{CR}$ networks. By using packet based channels, a major improvement observed in case of minimization of handoff delay. Our proposed mechanism is then compared to the handoff scheme with spectrum pool using a pre-emptive resume priority (PRP) M/G/1 improvement can be seen in the total service time as packet based handoff mechanism successfully minimizes the handoff delay with an queuing network model to analyze the total service time and based handoff delay in each case. Numerical and simulation results show significant improvement of approximately $20 \%$ with the increase in the PU arrival rate for packet as it guarantees faster service time for SUs.

\section{ACKNOWLEDGMENTS}

We hereby take this opportunity to express profound thanks and gratitude from the bottom of my hearts towards SVNIT, Surat and MITCOE for providing valuable platform and untiring encouragement during the preparation of this paper.

\section{REFERENCES}

[1] S. Haykin, "Cognitive Radio: Brain-Empowered Wireless Communication", IEEE JSAC, Feb 2005.Federal Communications Commission (FCC), "Notice of Proposed Rule Making," ET Docket no.04-113, May 25, 2004.

[2] Federal Communications Commission (FCC),"Notice of Proposed Rule Making," ET Docket no.06-156, October, 2006.

[3] Carlos Cordeiro, Kiran Challapali, Dagnachew Birru and Sai Shankar N, "IEEE 802.22: An Introduction to the First Wireless Standard based on Cognitive Radios", Journal of Communications, April 2006.

[4] IEEE 802.22 Working Group on Wireless Regional Area Network, http://www.ieee802.org/22/.
[5] E. Hossain , V. Bhargava., "Cognitive Wireless Communication Networks", Springer Science+ Business Media, 2007, LLC, ISBN 978-0-387-68830-5, e-ISBN 978-0-387-68832-9.

[6] J. Mitola, "Cognitive radio: Making software radio more personal” IEEE Pers. Comm. 2005, August 1999.

[7] Xiangwei Zhou, Jun Ma, Geoffrey Ye Li, Fellow, IEEE, Young Hoon Kwon and Anthony C. K. Soong, Senior Member, IEEE, Probability-Based Optimization of InterSensing Duration and Power Control in Cognitive Radio, IEEE transactions on wireless communication, Vol. 8, No. 10, Oct. 2009.

[8] Matteo Cesana, Francesca Cuomo, Eylem Ekici, "Routing in Cognitive Radio networks/; Challenges and solutions", Ad Hoc Networks.

[9] Yi Song and Jiang Xie, "Performance Analysis of Spectrum Handoff for Cognitive Radio Ad Hoc Networks without common control channel under Homogeneous Primary Traffic",IEEE INFOCOM 2011.

[10] Madeeha Aman, Saeed Mahfooz, Waheed Ur Rehman, "Handoff Delay in Cognitive Radios -A Concept Paper on Utilization of Guard Channels",978-1-61284-9416/11/2011 IEEE.

[11] Jiaqi DUAN, Yog LI, "An optimal spectrum Handoff Scheme for Cognitive Radio Mobile Ad Hoc Networks", Advances in Electrical and Computer Engineering, Volume 11,Number 3,2011.

[12] L-C. Wang and A. Chen, "On the Performance of Spectrum Handoff for Link Maintenance in Cognitive Radio", Wireless Pervasive Computing, 3rd International Symposium, 7 - 9 May 2008.

[13] Y. Zhang, "Spectrum Handoff in Cognitive Radio Networks: "Opportunistic and Negotiated Situations", IEEE International Conference on Communications, 2009.

[14] Han Han, Qihui Wu, Hong Yin, Spectrum Sensing for Real-time Spectrum Handoff in CRNs, 2010 3rd International Conference on Advanced Computer Theory and Engineering(ICA CTE).

[15] L-C. Wang and C-W. Wang, "Spectrum Handoff for Cognitive Radio Networks: Reactive-Sensing or Proactive-Sensing?", IEEE Performance, Computing and Communications Conference, December 2008.

[16] Madeeha Aman, Dr. Saeed Mahfooz, and Waheed Ur Rehman, "A Handoff using Guard Channels Scheme (HGCS) for Cognitive Radio Networks", Global Journal of Computer Science and Technology, Volume 11 Issue 20 Version 1.0 December 2011.

[17] John Paul Torregoza, Pham Ngoc Thai, Yun-Sop Han, Martin Andre, Fumio Teraoka, Hiroaki Harai and WonJoo Hwang, An Opportunistic Cross-Layer Architecture for New Generation Networks, IEEE Communications Society ,IEEE Globecom 2010 proceedings.

[18] Yi Song and Jiang Xie, Performance Analysis of Spectrum Handoff for Cognitive Radio Ad Hoc Networks without Common Control Channel under Homogeneous Primary Traffic, IEEE INFOCOM 2011. 